\title{
Levantamento taxonômico de desmídias (Chlorophyta) do lago Novo (Amapá, Brasil): Gêneros Staurastrum, Staurodesmus e Xanthidium
}

\author{
Karla Ferreira SOUZA ${ }^{1}$, Sergio MELO² \\ RESUMO \\ Neste estudo são descritas e ilustradas as espécies de três gêneros de desmídias (Staurastrum, Staurodesmus e Xanthidium) do \\ lago Novo, um ambiente localizado na região dos lagos, na planície costeira do Estado do Amapá. Um total de 35 táxons \\ foi registrado, sendo 23 do gênero Staurastrum, sete do gênero Staurodesmus e cinco do gênero Xanthidium. Todos os táxons \\ apresentados neste trabalho constituem primeira citação para o estado do Amapá.
}

PALAVRAS-CHAVE: Desmidiales, Região Amazônica, Taxonomia

\section{Desmids (Chlorophyta) from lake Novo (Amapá, Brazil): Genera Staurastrum, Staurodesmus e Xanthidium}

\section{ABSTRACT}

This study describes and sketches the species of three desmids genus (Staurastrum, Staurodesmus and Xanthidium) from Lake Novo, located in the coastal plain lake region of Amapá State. In total, 35 taxa were registered, being 23 of Staurastrum, seven of Staurodesmus and five of Xanthidium genus. These results are the first documented taxa registered in the Amapá State.

KEYWORDS: Desmidiales, Amazon region, Taxonomy 


\section{INTRODUÇÃO}

As desmídias, algas verdes da divisão Chlorophyta, classe Conjugatophyceae e das ordens Zygnematales e Desmidiales, são algas que têm preferência por ambientes de água doce, mas também ocorrem em ambientes salobros (Gerrath 1993). Estão entre as muitas espécies capazes de resistir a longos períodos de dissecaçáo e podem ser dispersas pelo vento ou por meio de animais que se movem de um corpo d'água para outro (Brook 1981). Em geral, espécies da ordem Desmidiales são encontradas em ambientes aquáticos oligo-mesotróficos (Coesel 1996; Gerrath 2003).

Esse grupo de algas tem uma distribuiçáo cosmopolita, apresentando, no entanto, um aumento no número de espécies em direção a linha do equador, resultando em uma flora tropical altamente diversificada (Coesel 1996). Destacase o fato de nas proximidades dos trópicos a temperatura da água apresentar valores próximos aos valores ótimos para o desenvolvimento destas algas, ou seja, em torno de $25-30^{\circ} \mathrm{C}$ (Coesel e Wardenaar 1990).

Muitas espécies de desmídias apresentam habitat perifítico, estando associadas à macrófitas aquáticas onde passam maior parte do seu ciclo de vida, no entanto, podem em determinado momento se desprender e tornar-se livre flutuante (Brook 1981; Coesel 1982). Os gêneros Staurastrum Meyen, Staurodesmus Teiling e Xanthidium Ehrenberg, inclusos na ordem Desmidiales, como a maioria dos gêneros de desmídias, podem ser encontrados tanto na coluna d'água como aderidos a substratos e apresentam uma grande diversidade taxonômica nos ambientes amazônicos, fato que pode ser constatado em vários trabalhos, tais como: Grönblad (1945); Förster (1963; 1964; 1969; 1974); Scott et al. (1965); Thomasson (1971); Sophia e Huszar (1996); Lopes e Bicudo (2003); Melo e Souza (2009).

Embora as desmídias constituam um dos grupos de algas melhor conhecido taxonomicamente na região amazônica, os trabalhos estáo concentrados principalmente nos estados do Pará e Amazonas, com base em amostras de poucos ambientes (Lopes e Bicudo 2003; Melo et al. 2005). Para o estado do Amapá, cita-se apenas o trabalho realizado por Förster (1963), no qual o autor cita cinco táxons de desmídias (Desmidium laticeps Nordstedt fac. 4-radiata, Desmidium laticeps Nordstedt fac. 5-radiata, Euastrum laticeps f. evolutum, Cosmarium pseudomagnificum f. brasilense e Closterium pseudolunula var. concavum, sendo as duas últimas espécies, uma nova forma $\mathrm{e}$ nova variedade, respectivamente).

Visando ampliar o conhecimento sobre a diversidade taxonômica das desmídias da Amazônia, foi realizado o presente estudo que teve por objetivos identificar, descrever e ilustrar as espécies de desmídias dos gêneros Staurastrum Meyen, Staurodesmus Teiling e Xanthidium Ehrenberg de habitat planctônico de um lago da região dos lagos, no Estado do Amapá

\section{MATERIAL E MÉTODOS}

Este estudo foi conduzido a partir de amostras coletadas no lago Novo, um ambiente raso e ligeiramente ácido, localizado na regiáo dos lagos na planície costeira do Estado do Amapá, em uma área limite com a Reserva Biológica do Lago Piratuba, região denominada de cinturão lacustre Meridional, de acordo com Silveira (1998). A regiáo apresenta clima caracterizado por elevada precipitaçáo anual, variando em média entre 1.500 e $3.500 \mathrm{~mm}$, com maiores índices pluviométricos concentrados nos meses de dezembro a maio, e com temperatura média de $27^{\circ} \mathrm{C}$ (Dias 2007).

Para este estudo, as amostras foram coletadas no mês de junho de 2005, em nove estaçóes na região pelágica. Para tanto, foi utilizada rede de plâncton com abertura de malha de $25 \mu \mathrm{m}$, e o material coletado foi fixado com solução Transeau (Bicudo e Menezes 2006). Todo material foi depositado no Herbário do Instituto Nacional de Pesquisas da Amazônia, sob número de registro de INPA 223918-223930.

Os números de registro no Herbário, bem como as coordenadas geográficas de cada estação de coleta, os valores referentes à profundidade, transparência, temperatura, $\mathrm{pH} \mathrm{e}$ condutividade elétrica da água são apresentados na Tabela 1.

A análise das amostras foi realizada em microscópio óptico, equipado com câmara clara, e a identificaçáo dos táxons foi feita considerando os caracteres morfológicos e morfométricos. $\mathrm{O}$ arranjo taxonômico foi baseado no sistema proposto por Mix (1972). A identificação dos táxons foi baseada em literatura específica como: Grönblad (1945), Scott et al. (1965), Förster (1969; 1974), Teiling (1967), Thomasson (1971), Croasdale et al. (1994).

\section{RESULTADOS E DISCUSSÃO}

A descrição e ilustração dos táxons registrados são apresentadas a seguir, seguindo exclusivamente a ordem alfabética. Além disso, acompanha a descrição a informação sobre o local de ocorrência do táxon, cujas características limnológicas e a coordenada geográfica são apresentadas na Tabela 1.

1- Staurastrum boergensenii Raciborski var. elegans Borge, Arkiv Bot. 19 (17): 1-56.1925. (Figura 1- Estampa 1)

Células $1,1-1,3$ vezes mais longas que largas; 76-123 x 89-153 $\mu \mathrm{m}$ (com processo); 38-60 x 28-43 $\mu \mathrm{m}$ (sem processo); istmo 18-33 $\mu \mathrm{m}$. Semicélula copuliforme; ângulos prolongados em processos cilíndricos, divergentes, longos e lisos, terminando com dois espinhos na extremidade; margem apical convexa com espinhos curtos bifurcados; fileira de espinhos na regiáo istimal. Vista apical náo observada. 
Tabela 1 - Dados dos pontos de amostragem no lago Novo (AP) durante o período de cheia de 2005 com coordenadas e variáveis abióticas (Prof. = Profundidade; Secchi = transparência da água; $\mathrm{pH} ; \mathrm{CE}=$ condutividade elétrica da água; Temp. = Temperatura).

\begin{tabular}{|c|c|c|c|c|c|c|c|c|}
\hline \multirow{2}{*}{ Estação } & \multirow{2}{*}{ № Herbário } & \multicolumn{2}{|c|}{ Coordenadas } & \multirow{2}{*}{$\begin{array}{c}\text { Prof. } \\
\text { (metros) }\end{array}$} & \multirow{2}{*}{$\begin{array}{l}\text { Secchi } \\
\text { (metros) }\end{array}$} & \multirow{2}{*}{$\mathrm{pH}$} & \multirow{2}{*}{ CE $\left(\mu \mathrm{S} . \mathrm{cm}^{-1}\right)$} & \multirow{2}{*}{ Temp $\left({ }^{\circ} \mathrm{C}\right)$} \\
\hline & & $S$ & W & & & & & \\
\hline 1 & INPA 223918 & $1^{0} 33^{`} 51.41^{\prime \prime}$ & $50^{\circ} 42^{` 30.64 ”}$ & 2,50 & 1,55 & 5,13 & 28 & 28,2 \\
\hline 2 & INPA 223919 & 1034 08.80” & $50^{\circ} 42^{`} 59.61^{\prime \prime}$ & 2,30 & 1,15 & 5,53 & 24 & 28,8 \\
\hline 3 & INPA 223921 & $1^{0} 32 ` 18.72 ”$ & $50^{\circ} 41^{` 08.07 ”}$ & 2,00 & 0,85 & 5,55 & 37 & 28,7 \\
\hline 4 & INPA 223922 & $1^{0} 29^{\circ} 01.83^{\prime \prime}$ & $50^{\circ} 40^{`} 31.86^{\prime \prime}$ & 2,60 & 0,51 & 5,52 & 38 & 29,3 \\
\hline 5 & INPA 223924 & $1^{0} 28 ` 47.35^{\prime \prime}$ & $50^{\circ} 37^{`} 46.77^{\prime \prime}$ & 2,15 & 0,40 & 5,86 & 118 & 29,9 \\
\hline 6 & INPA 223926 & $1^{0} 25 ` 26.12^{\prime \prime}$ & $50^{\circ} 41^{`} 44.26^{\prime \prime}$ & 2,60 & 1,85 & 5,00 & 18 & 30,0 \\
\hline 7 & INPA 223927 & $1^{0} 23 ` 17.24^{\prime \prime}$ & $50^{\circ} 38^{`} 47.58^{\prime \prime}$ & 2,60 & 0,65 & 5,54 & 23 & 30,2 \\
\hline 8 & INPA 223929 & $1^{0} 26 ` 12.44^{\prime \prime}$ & $50^{\circ} 35^{`} 55.23^{\prime \prime}$ & 2,30 & 0,55 & 5,31 & 29 & 29,7 \\
\hline 9 & INPA 223930 & $1^{0} 34^{\prime} 10.25 "$ & $50^{\circ} 43^{`} 15.54 ”$ & - & - & - & - & - \\
\hline
\end{tabular}

\section{Ocorrência: Estação 6}

2- Staurastrum boergensenii Raiborski var. scotii Förster, Amazoniana 2: 78, Pl. 48, Fig. 3. 1969. (Figura 1- Estampas 2-3)

Células 1,4-1,7 vezes mais longas que largas; 56-70 x 82-115 $\mu \mathrm{m}$ (com processo); 39-46 x 30-40 $\mu \mathrm{m}$ (sem processo); istmo $18-30 \mu \mathrm{m}$. Semicélula copuliforme; ângulos prolongados em processos cilíndricos, divergentes, lisos, com dois espinhos na extremidade; margem apical convexa com espinhos curtos bifurcados. Vista apical 7-angular; margem côncava, espinhos bifurcados submarginais.

Ocorrência: Estação 6

3- Staurastrum brachiatum Ralfs, Brit. Desm. p.131. Pl. 23. Fig. 9. 1848. (Figura 1- Estampa 4)

Células 1,1 -1,2 mais longas que largas; 43-60 x 50-72 $\mu \mathrm{m}$ (com processo); 23-35 x 15-20 $\mu \mathrm{m}$ (sem processo); istmo 8-18 $\mu \mathrm{m}$. Semicélula quadrangular; seno mediano ligeiramente aberto; ângulos prolongados em processos subcilíndricos, lisos e divergentes nos ângulos com extremidades indentadas; margem apical côncava; margem lateral reta ou ligeiramente convexa. Vista apical não observada.

Ocorrência: Estaçóes 1, 6 e 8

4- Staurastrum brasiliense Nordestedt var. porrectum Borge, Arkiv Bot. p. 39. Pl. 5, Fig. 16. 1925. (Figura 1- Estampas 5-6)

Células tão longas quanto largas até 1,1 vezes mais longas que largas; vezes mais longas que largas; 76-136 x 76-126 $\mu \mathrm{m}$ (com espinhos); 46-76 x 46-59 $\mu \mathrm{m}$ (sem espinho); istmo $20-31 \mu \mathrm{m}$. Semicélula copuliforme; margem apical reta ou ligeiramente convexa; margem lateral convexa; ângulos terminados com três espinhos robustos divergentes. Vista apical 5-angular; margens lisas e côncavas.

\section{Ocorrência: Estações 3 e 4}

5- Staurastrum brebissonii Archer var. brasiliense Grönblad, Acta Soc. Sci. Fenicae. p. 24. Pl. 9. Fig. 198. 1945. (Figura 1- Estampas 7-8)

Células 0,9-1,1 mais longas que largas, 52-78 x 86-100 $\mu \mathrm{m}$ (com espinhos), 42-62 x 40-59 $\mu \mathrm{m}$ (sem espinhos); istmo 15-23 $\mu \mathrm{m}$. Semicélula triangular; seno mediano aberto; parede celular com espinhos em toda semicélula formando anéis concêntricos; margem apical e lateral convexa; seno mediano aberto. Vista apical 3-angular com espinhos marginais e submarginais.

Ocorrência: Estaçôes 4, 5, 7 e 9

6-Staurastrum dispergens Grönblad, Acta Soc. Sci. Fenicae. p. 25. Pl. 10, Fig. 206. 1945. (Figura 1- Estampa 9)

Células 1,8 vezes mais longa que larga; 40-41 x 22-35 $\mu \mathrm{m}$ (com espinho); 20-25 x 14-18 $\mu \mathrm{m}$ (sem espinho); istmo 6-8 $\mu \mathrm{m}$. Semicélula hexagonal; seno mediano aberto; ângulos prolongados em um par de espinhos divergentes; margem apical reta; fileira de espinhos na região apical e na regiáo basal da semicélula. Vista apical náo observada.

\section{Ocorrência: Estação 1}

7- Staurastrum elegantissimum Johnson var. brasiliense Förster, Amazoniana. p. 81, Pl. 46, Figs. 1-3. 1969. (Figura 1- Estampas 10-14)

Células 1,4 - 1,6 vezes mais longas que largas; 35-55 x 50-86 $\mu \mathrm{m}$ (com processo); 25-30 $\mu \mathrm{m}$ (largura sem processo); istmo 12-17 $\mu \mathrm{m}$. Semicélula obtrapeziforme; seno mediano aberto; margem apical reta ou ligeiramente convexa; processos com margens denticuladas terminando com espinhos na extremidade; verrugas marginais e submarginais; espinhos na 
regiāo istimal. Vista apical 5-angular, forma dicotípica em um mesmo espécime, vista apical 3-angular e 5-angular.

Ocorrência: Estaçóes 1, 2, 3, 4, 7 e 9

8- Staurastrum gladiosum Turner f. ornata Laporte, Encyclop. Biol.; 9:118. Pl. 15, Figs. 177-179. (Figura 1Estampas 15-16)

Células 1,1-1,3 vezes mais longas que largas; 37-46 x 35-38 $\mu \mathrm{m}$ (com espinho); 30-37 x 25-28 $\mu \mathrm{m}$ (sem espinho); istmo $12-15 \mu \mathrm{m}$. Semicélula elíptica com margens lateral e apical convexa margeada com espinhos curtos; duas fileiras de espinhos na face da semicélula; vista apical 3-angular com espinhos marginais e submarginais.

Ocorrência: Estação 1

9- Staurastrum grallatorium Nordestedt var. brasiliense (Grönblad) Förster, Amazoniana. p. 83, Pl. 39, Fig.3. 1969. (Figura 1- Estampa 17)

Células 2,1-2,2 mais larga que longa; 35-51 x 74-117 $\mu \mathrm{m}$ (com processo); $25-43 \mu \mathrm{m}$ (largura sem processo); istmo 10-12 $\mu \mathrm{m}$. Semicélula campanular; margem apical reta com espinhos bifurcados; ângulos prolongados em processos horizontais com margens serreadas terminando com dois espinhos robustos na extremidade. Vista apical não observada.

\section{Ocorrência: Estações 1e 3}

10- Staurastrum leptacanthum Nordestedt, Vid. Medd. Naturh. Foren. Kjöbenhavn. p. 229. P1. 4. Fig.46. 1870. (Figura 1- Estampas 18-19)

Células 1,0-1,1 tão longas quanto largas; 42-58 x 42-52 $\mu \mathrm{m}$ (com processo); $25-51 \times 11-18 \mu \mathrm{m}$ (sem processo); istmo 4-11 $\mu \mathrm{m}$. Semicélula arredondada, margens lateral e apical convexas; ângulos prolongados em processos cilíndricos, divergentes e lisos com dois dentículos na extremidade. Vista apical 9-angular.

\section{Ocorrência: Estaçóes 2,3 e 4}

11 - Staurastrum novae-cesareae Wolle var. brasiliense (Grönblad) Förster, Amazoniana. p. 89, Pl.34, Figs. 5-9. 1969. (Figura 1- Estampas 20-21)

Células 1,2-1,6 vezes mais largas que longas; 48-87 x 58-83 $\mu \mathrm{m}$ (com espinho); 35-62 x 31-56 $\mu \mathrm{m}$ (sem espinho); istmo 15-25 $\mu \mathrm{m}$. Semicélula transversalmente elíptica; grânulos distribuídos em toda a semicélula; ângulos terminados com espinhos simples e divergentes; margem apical e lateral convexa. Vista apical 4-angular com margens retas e com verrugas marginais e submarginais.

Ocorrência: Estação 1

12-Staurastrum nudibrachiatum Borge, Arkiv Bot. p. 109, Pl. 4, Fig. 20, 1903. (Figura 1- Estampa 22)

Célula tão longas quanto largas até 1,1 vezes mais largas que longas; $56-67$ x 56-72 $\mu \mathrm{m}$ (com processo); 40-44 x 23-35 $\mu \mathrm{m}$ (sem processo); istmo 20-22 $\mu \mathrm{m}$. Semicélula arredondada; seno mediano agudo; istmo largo; margem apical e basal convexa; ângulos prolongados em processos lisos e divergentes. Vista apical não observada.

Ocorrência: Estação 7

13- Staurastrum paradoxum Meyen in Ralfs, British Desmidiaceae. p. 138, Pl. 23, Fig. 8. 1848. (Figura 1- Estampas 23-24)

Células 1,1 vezes mais largas que longas; 26-34 x 22-38 $\mu \mathrm{m}$ (com processo); $18-20 \times 14-18 \mu \mathrm{m}$ (sem processo); istmo 5-8 $\mu \mathrm{m}$. Semicélula subtriangular; seno mediano agudo; margem apical reta ou ligeiramente côncava; processos divergentes com margens serreadas terminando com dentículos na extremidade. Vista apical 3-angular com lados retos e lisos.

Ocorrência: Estação: 7

14- Staurastrum quadrangulare Brébisson ex Ralfs var. longispinum Börgesen, Vidensk. Medd. Naturh. Forein. Kjobenhavn, 34, Pl.5, Fig. 55. 1890. (Figura 1- Estampas 25-26)

Células 1,1-1,2 vezes mais longas que largas; 21-36 x 26-40 $\mu \mathrm{m}$ (com espinho); 15-26 x 17-34 $\mu \mathrm{m}$ (sem espinho); istmo 5-14 $\mu \mathrm{m}$. Semicélula retangular, margem apical reta ou ligeiramente côncava; um espinho na região acima do istmo; ângulos terminados com espinhos divergentes; vista apical 3-angular com lados côncavos.

Ocorrência: Estações 3, 7, 8 e 9

15- Staurastrum quadrangulare Brébisson ex Ralfs var. prolificum Croasdale in Scott, Grönblad e Croasdale, Acta Botanica Fennica. p. 69, Pl.12, Figs:176-177 1965. (Figura 1 - Estampas 27-28)

Células até 1,1 vezes mais largas que longas; 27-37 x 27-40 $\mu \mathrm{m}$ (com espinho); 25-27 x 20-30 $\mu \mathrm{m}$ (sem espinho); istmo 10-16 $\mu \mathrm{m}$. Semicélula quadrangular; margem apical reta ou ligeiramente côncava; margem lateral convexa; ângulos prolongados em espinhos curtos; região acima do istmo com espinhos bifurcados; vista apical 4-angular.

Ocorrência: Estaçôes 6 e 7

16- Staurastrum rotula Nordestedt, Vidensk. Medd. Naturh. Forein. Kjöbenhavn, p. 227, Pl. 4, Fig. 38. 1870. (Figura 1Estampas 29-30)

Células 1,6-2,1 vezes mais largas que longas; 32-50 x 66-80 $\mu \mathrm{m}$ (com processo); 13-18 $\mu \mathrm{m}$ (largura sem processo); istmo 5-10 $\mu \mathrm{m}$. Semicélula subhexagonal; constrição mediana profunda; ângulos prolongados em processos retos ou ligeiramente convergentes com espinhos na extremidade; margem dos processos serreados; vista apical 7-angular.

\section{Ocorrência: Estação 7}


17- Staurastrum sebaldii Reinsch var. ornatum f. planctonica (Lütkem.)Teiling, Svensk Bot. Tidskr. p. 226. Figs. 12,14. 1947. (Figura 1- Estampas 31-32).

Células 1,7-2,0 vezes mais largas que longas; 40-66 x 83$115 \mu \mathrm{m}$ (com processo); 23-35 $\mu \mathrm{m}$ (largura sem processo); istmo 10-20 $\mu \mathrm{m}$. Semicélula obtrapeziforme; ângulos prolongados em processos retos com margens serreadas com dentículos na extremidade; fileira de verrugas na região istmial; vista apical 3-angular.

Ocorrência: Estação 7

18-Staurastrum setigerum Cleve var. occidentale West e West, Trans. Linn. Soc. Lond, p. 260, pl. 16, fig. 28. 1896 (Figura 2- Estampas 33-34)

Células 0,9-1,1 mais largas que longas; 30-38 x 28-42 $\mu \mathrm{m}$ (com espinho); $18-30 \times 20-25 \mu \mathrm{m}$ (sem espinho), istmo 4-8 $\mu \mathrm{m}$. Semicélula triangular com margem lateral convexa e margem apical reta ou ligeiramente convexa, com espinhos marginais; seno mediano agudo. Vista apical 3-angular.

\section{Ocorrência: Estaçâo 7}

19- Staurastrum setigerum Gleve var. pectinatum West e West, Trans. Linm. Soc. London, Bot., p. 260. Pl. 16, Fig. 28. 1896. (Figura 2- Estampas 35-36)

Células $0,8-1,2$ vezes mais longas que largas; $50-76$ × 76-90 $\mu \mathrm{m}$ (com espinho); 40-60 x 42-75 $\mu \mathrm{m}$ (sem espinho); istmo 10-14 $\mu \mathrm{m}$. Semicélula triangular com margem apical convexa e espinhos marginais, margem lateral lisa e convexa; seno mediano aberto; face da semicélula com espinhos concêntricos na região mediana; vista apical 3-angular.

Ocorrência: Estaçóes 2, 6 e 7

20- Staurastrum setigerum Gleve var. tristichum Nygaard f. furcatum Förster, Amazoniana. p. 94. Pl. 34, Fig. 2. 1969. (Figura 2- Estampa 37)

Células 0,8-1,2 vezes mais longas que largas; 46-65 x 50-80 $\mu \mathrm{m}$ (com espinho); 40-48 x 34-39 $\mu \mathrm{m}$ (sem espinho); istmo 11-13 $\mu \mathrm{m}$. Semicélula triangular; margem apical convexa com espinhos marginais; face da semicélula decorada na regiāo mediana com um par de espinhos robustos; margem lateral convexa e lisa; dois espinhos robustos e divergentes nos ângulos. Vista apical não observada.

\section{Ocorrência: Estação 1}

21- Staurastrum stelliferum Borge var. stelliferum, Arkiv Bot. p. 42, Pl.6, Fig. 3. 1925. (Figura 2- Estampas 38-41)

Células tão longas quanto largas, até 1,1 vez; 51-87 x 59$101 \mu \mathrm{m}$ (com processo); $28-38 \times 17-30 \mu \mathrm{m}$ (sem processo); istmo 8-15 $\mu \mathrm{m}$. Semicélula obtrapeziforme; margem apical reta; margem lateral ligeiramente convexa; seno mediano aberto; ângulos prolongados em processos lisos, cilíndricos e divergentes com dentículos na extremidade e na base; vista apical 4/5-angular; lados retos e lisos.

\section{Ocorrência: Estaçôes 6, 8, 9}

22- Staurastrum tectum Borge var. ayayense Grönblad, Acta Soc. Sci. Fennica p. 31, Pl. 14, Figs. 289-291. 1945. (Figura 2- Estampas 42-43)

Células 1,3-1,4 vezes mais larga que longa que longa; 51-67 x 76-110 $\mu \mathrm{m}$ (com processo); $21-42 \times 14-28 \mu \mathrm{m}$ (sem processo); istmo $5-15 \mu \mathrm{m}$; semicélula triangular; margem apical reta com espinhos bifurcados; ângulos prolongados em processos serreados e divergentes com extremidades denticuladas; presença de espinhos na região central da semicélula; vista apical 2-angular.

\section{Ocorrência: Estação 7}

23- Staurastrum trifidum Nordstedt var. inflexum West e West, Trans. Linn. Soc. Lond. Bot.; p. 258, Pl. 16, Fig. 22. 1896. (Figura 2- Estampas 44-45)

Células 1,2-1,4 vezes mais largas que longas; 37 x 46-52 $\mu \mathrm{m}$ (com espinho); 30-37 $\mu \mathrm{m}$ (largura sem); istmo 12-15 $\mu \mathrm{m}$. Semicélula triangular; margem apical convexa; margem lateral reta; espinhos curtos e convergentes nos ângulos; vista apical 3- angular com lados retos e lisos.

Ocorrência: Estações 4, 7, 8

24-Staurodesmus clepsidra (Nordestedt) Teiling var. obtusus (Nordestedt) Teiling, Arkiv Bot. p. 495. Pl.2, Fig. 9. 1967. (Figura 2- Estampas 46-47)

Células 1,1-1,2 vezes mais largas que longas; 21-28 x 24-32 $\mu \mathrm{m}$; istmo 5-12 $\mu \mathrm{m}$; semicélula triangular; ângulos arredondados e espessados; seno mediano agudo; margem apical reta. Vista apical 3-angular.

Ocorrência: Estação 6

25- Staurodesmus cuspidatus (Brébisson) Teiling var. groenbladii Förster, Amazoniana. p. 67, Pl.29, Figs.1819.1969. (Figura 2- Estampas 48-49)

Células tão longas quanto largas até 1,1 vezes mais largas que longas; $25-33$ x 25-35 $\mu \mathrm{m}$ (com espinho); 13-15 x 10-17 $\mu \mathrm{m}$ (sem espinho); istmo 5-7 $\mu \mathrm{m}$. Semicélula triangular; seno mediano agudo, istmo aberto; margem apical côncava; ângulos prolongados em espinhos divergentes. Vista apical 3-angular.

Ocorrência: Estação 6

26- Staurodesmus cf. convergens (Ehrenberg) Teiling, Bot. Notiser, p. 587, Pl. 25: 1,2; Pl. 26, Fig. 3. (Figura 2- Estampa 50)

Células 1,5-1,6 vezes mais largas que longas; 33-40 x 53-62 $\mu \mathrm{m}$ (com espinho); 39-47 $\mu \mathrm{m}$ (largura sem espinho); istmo 4-10 $\mu \mathrm{m}$. Semicélulas fusiformes; seno mediano agudo; istmo aberto; margem apical convexa; ângulos prolongados em espinhos convergentes. Vista apical não observada (por esse motivo colocamos a espécie com conferatur, pois para a identificação da espécie é necessária observação da vista apical). 


\section{Ocorrência: Estaçóes 4 e 8}

27- Staurodesmus extensus (Borge) Teiling var. extensus Teiling, p. 67, Fig. 11. 1948.

(Figura 2- Estampas 51-52)

Células 1,3 vezes mais largas que longas; 23-43 x 30$56 \mu \mathrm{m}$; 16-20 $\mu \mathrm{m}$ (largura sem espinho); istmo 4-7 $\mu \mathrm{m}$. Semicélula triangular; seno mediano aberto e arredondado; margem apical reta; margem lateral ondulada, ângulos prolongados em espinhos longos e paralelos.

Ocorrência: Estação 1, 3 e 7

28-Staurodesmus glaber (Ehrenberg) Teiling var. flexispinus (Förster e Eckert) Teiling, Arkiv Bot. p. 559, Pl. 13, Fig. 17. 1967. (Figura 2- Estampas 53-54)

Células 2,1-2,3 vezes mais largas que longas; 33-45 x 77-95 $\mu \mathrm{m}$ (com espinho); 34-50 $\mu \mathrm{m}$ (largura sem espinho); istmo 13$25 \mu \mathrm{m}$. Semicélula triangular; seno mediano agudo; margem apical reta; ângulos prolongados em espinhos paralelos e ligeiramente curvados; vista apical 3- angular.

Ocorrência: Estação 6

29- Staurodesmus triangularis (Lagerheim) Teiling var. triangularis Teiling, Bot. Notiser. p. 62, Figs. 63-68. (Figura 2- Estampa 56).

Células 2,2 vezes mais largas que longa; 35 x $80 \mu \mathrm{m} ; 23$ $\mu \mathrm{m}$ (largura sem espinho); istmo $7 \mu \mathrm{m}$. Semicélula triangular; seno mediano aberto e arredondado; margem apical e lateral reta; ângulos prolongados em espinhos longos e paralelos. Vista apical não observada.

\section{Ocorrência: Estação 7}

30- Staurodesmus validus (West e West) Thomasson var. subvalidus (Grönblad) Teiling, Ark. Bot. p. 566, Pl. 11, Fig. 11. 1967. (Figura 2- Estampa 55)

Células tão longas quanto largas até 1,2 vezes mais longas que largas; $57-88$ x 47-90 $\mu \mathrm{m}$ (com espinho); 26-45 x 25-50 $\mu \mathrm{m}$ (sem espinho); istmo 5-10 $\mu \mathrm{m}$; semicélula triangular; seno mediano aberto e arredondado; ângulos prolongados em espinhos divergentes; margem apical côncava; dois pirenóides em cada semicélula. Vista apical não observada.

\section{Ocorrência: Estação 1}

31- Xanthidium canadense (Joshua) Förster var. magnum Förster, Amazoniana, p. 60, Pl. 23, Fig. 1. 1969. (Figura 2- Estampa 57)

Células 1,1-1,2 vezes mais largas que longas; 140-190 x 166- $225 \mu \mathrm{m}$ (com espinho; 83-120 $\mu \mathrm{m}$ x 86-110 $\mu \mathrm{m}$ (sem espinho); istmo 36-50 $\mu \mathrm{m}$. Semicélula elíptica; seno mediano fechado, face da semicélula lisa; margem apical convexa; ângulos prolongados em espinhos divergentes. Vista apical não observada.

Ocorrência: Estaçôes 4 e 9

32- Xanthidium fragile Borge K. Sv. Vet. - Akad. Hel. p. 17, Pl. 1, Fig. 14. 1899. (Figura 2- Estampa 58)

Células 1,1-1,3 vezes mais largas que longas; 88-123 x 75-100 $\mu \mathrm{m}$ (com espinho); 66-97 x 43-65 $\mu \mathrm{m}$ (sem espinho); istmo 21-30 $\mu \mathrm{m}$. Semicélula hexagonal; margem apical côncava; ângulos prolongados em espinhos curtos e bifurcados; face da semicélula decorada com espinhos simples, divergentes e robustos. Vista apical não observada.

\section{Ocorrência: Estação 1}

33-Xanthidium mamillosum (Grönblad) Förster var. borgei Förster, Hydrobiologia. p.411, Pl. 26, Fig. 1. 1964. (Figura 2- Estampas 59-60)

Células 1,1-1,3 vezes mais largas que longas; 52-60 x 60-80 $\mu \mathrm{m}$ (com espinho); $35-42 \times 23-50 \mu \mathrm{m}$ (sem espinho); istmo $15 \mu \mathrm{m}$. Semicélula oval; seno mediano agudo; margem apical reta; ângulos prolongados em espinhos divergentes; vista apical 4-angular.

Ocorrência: Estaçóes 1, 3 e 6

34- Xanthidium mamillosum (Grönblad) Förster var. longispinum (Borge) Förster, Amazoniana. p. 162. Pl.9 Fig.1. 1974. (Figura 2- Estampas 61-62)

Células 1,3-1,5 vezes mais largas que longas; 64-96 x 98$126 \mu \mathrm{m}$ (com espinho); 38-46 x 19-33 $\mu \mathrm{m}$ (sem espinho); istmo $20 \mu \mathrm{m}$; semicélula elíptica; seno mediano agudo; margem apical côncava; ângulos prolongados em um par de espinhos divergentes; vista apical 2-angular.

\section{Ocorrência: Estação 6}

35- Xanthidium trilobum Nordstedt Vidensk. Meddr. Dansk naturh. Foren., p. 230. Pl. 3, Fig. 35 a-c. 1869. (Figura 2- Estampa 63)

Células tão longas quanto largas até 1,1 mais longas que largas; 107-113 x 90-116 $\mu \mathrm{m}$ (com espinho); 70-75 x 67-83 $\mu \mathrm{m}$ (sem espinho); istmo 20-26 $\mu \mathrm{m}$. Semicélula hexagonal; seno mediano aberto e arredondado; ângulos prolongados em espinhos e bifurcados; face da semicélula decorada com espinhos concêntricos na regiáo mediana. Vista apical náo observada.

Ocorrência: Estação 6 

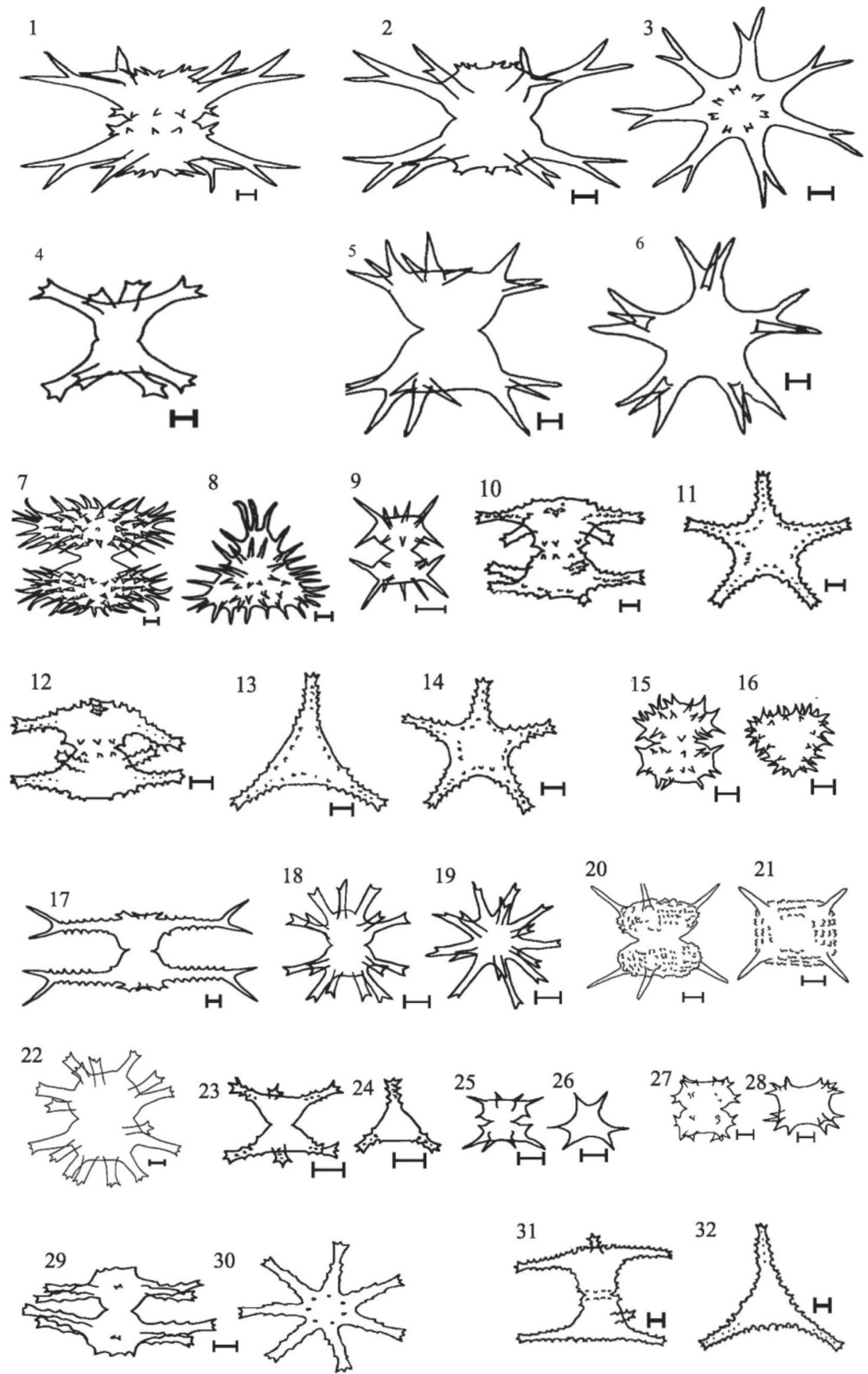

Figura 1 - Representação esquematizada de desmídias encontradas no lago Novo- AP. 1- S. boergensenii var. elegans; 2,3- S. boergensenii var. scotii; 4- S. brachiatum; 5,6- S. brasiliense var. porrectum; 7,8- S. brebissonii var. brasiliense; 9- S. dispergens; 10,11- S. elegantissimum var. brasiliense; 12-14- S. elegantissimum var. brasiliense forma dicotípica; 15, 16- $S$. gladiosum f. ornata; 17- S. grallatorium var. brasiliense; 18,19- $S$. leptacanthum; 20, 21- $S$. novae-cesareae var. brasiliense; 22-S. nudibrachiatum; 23, 24- S. paradoxum; 25, 26- S. quadrangulare var. longispinum; 27, 28- S. quadrangulare var. prolificum; 29,30- S. rotula; 31, 32-S. sebaldii var. ornatum f. planctônica. Escala $=10 \mu \mathrm{m}$. 

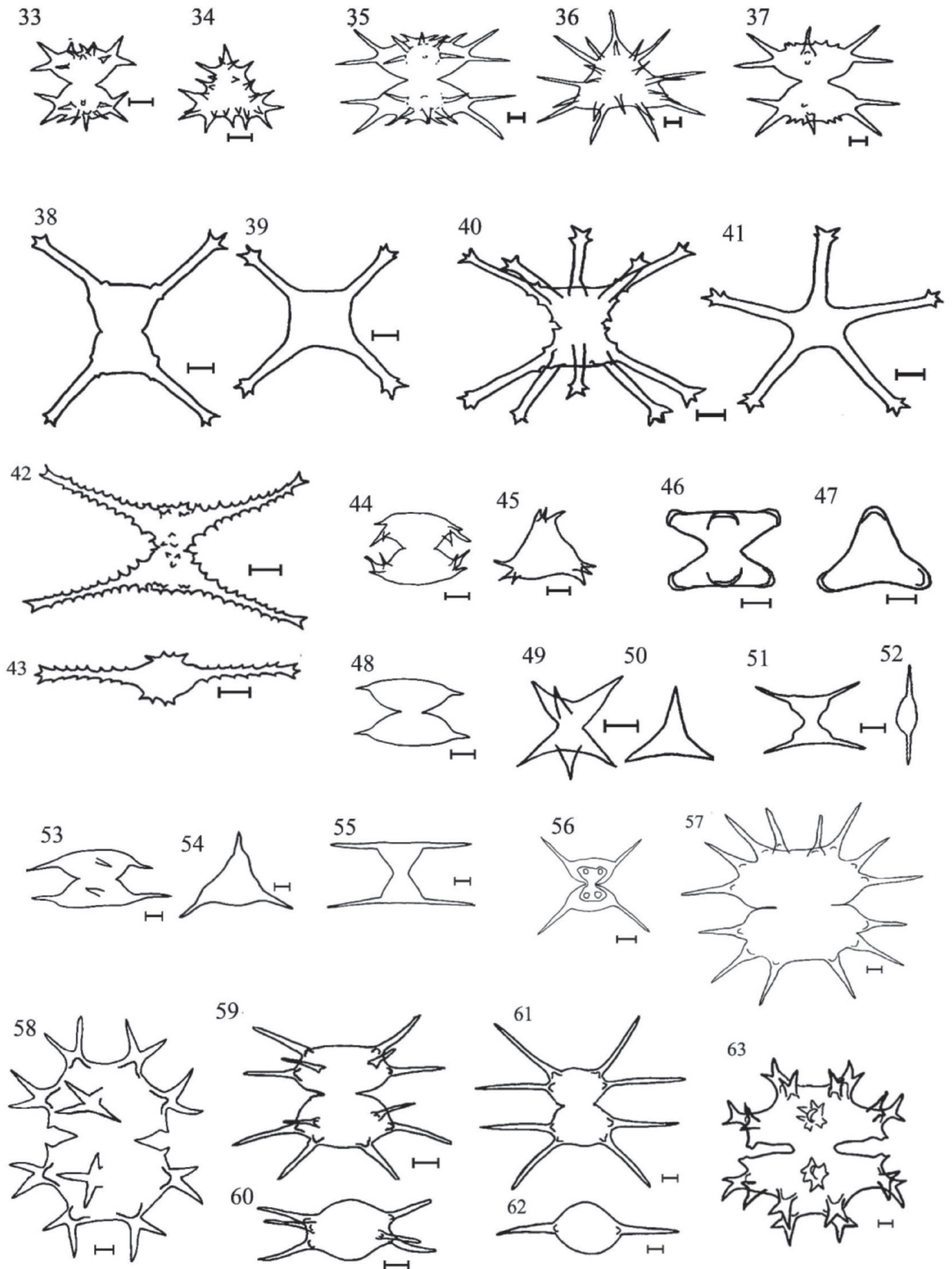

59

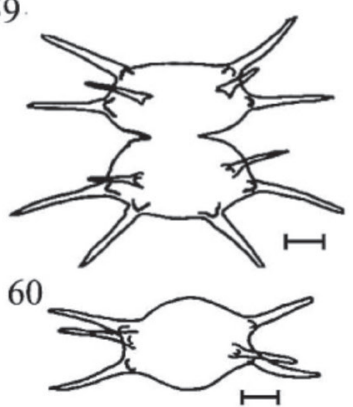

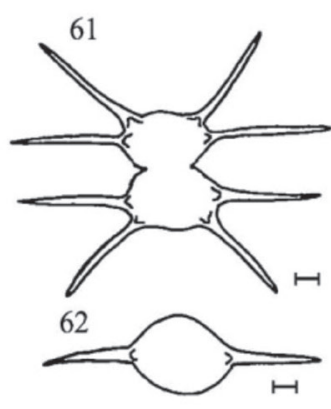

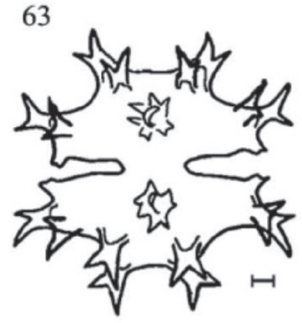

Figura 2 - Representação esquematizada de desmídias encontradas no lago Novo- AP. 33,34- S. setigerum var. occidentale; 35-36- S. setigerum var. pectinatum; 37- S. setigerum var. tristichum f. furcatum. 38-41- S. stelliferum var. stelliferum; 42-43- S. tectum var. ayayense; 44-45- S. trifidum var. inflexum; 46-47Staurodesmus clepsidra var. obtusus; 48- St. cf. convergens ; 49, 50- St. cuspidatus var. groenbladii;; 51-52- St. extensus var. estensus; 53-54- St. glaber var. flexispinus; 55- St. triangularis var. triangularis; 56- St. validus var. subvalidus; 57- Xanthidium canadense var. magnum; 58- Xanthidium fragile; 59-60-X. mamillosum var. borgei; 61-62-X. mamillosum var. Iongispinum; $63-X$. trilobum. Escala $=10 \mu \mathrm{m}$. 


\section{CONSIDERAÇÕES FINAIS}

O conhecimento da biodiversidade das algas de águas doces, continentais amazônicas, se restringe a poucas regióes, principalmente, aos estados do Amazonas e do Pará. Até o momento, por exemplo, cita-se apenas o trabalho de Förster (1963) para a região do rio Oyapoch no estado do Amapá abordando-as. O presente estudo apresenta resultados pioneiros neste sentido, a partir do registro e da descrição de 35 espécies de desmídias (23 espécies de Staurastrum, 7 Staurodesmus e 5 Xanthidium).

A grande riqueza de espécies de desmídias, dos gêneros estudados, pode estar associada ao fato de as estaçóes de coletas apresentarem baixa profundidade, baixa condutividade elétrica, exceto a estaçáo 5 , com valores de $\mathrm{pH}$ entre 5,00 e 5,86, e temperatura média de $29,4^{\circ} \mathrm{C}$, como pode ser observado na Tabela 1 . Tal proposiçáo se baseia no fato de este grupo de algas estar associado a ambientes com tais características (Melo e Souza 2009).
Outro fator que pode estar associado a esta elevada diversidade de desmídias na área estudada é a presença dos extensos bancos de macrófitas aquáticas ao longo do lago, facilitando a associação do grupo a estes bancos , que são posteriormente carreados para a coluna d'água pela ação da turbulência (Brook 1981; Happey-Wood 1988), turbulência esta que pode ter sido favorecida pela pequena profundidade.

$\mathrm{Na}$ Tabela 2 são apresentadas as espécies registradas, bem como a estação de ocorrência das mesmas. Staurastrum elegantissimum Johnson var. brasiliense Förster, Staurastrum brebissonii Archer var. brasiliense Grönblad e Staurastrum quadrangulare Brébisson var. longispinum Börgesen foram os táxons mais comuns, estando o primeiro presente em $56 \%$ das amostras analisadas e os seguintes em $44 \%$. Por outro lado, desperta a atenção que $58 \%$ dos táxons foram restritos a uma única estação de coleta.

Tabela 2 - Lista de espécies e ocorrência nas diferentes estações amostrais (E1, E2, E3, E4, E5, E6, E7, E8, E9) no lago Novo (AP) durante o período de cheia de 2005.

\begin{tabular}{|c|c|c|c|c|c|c|c|c|c|}
\hline Espécies/estações de coletas & $\mathrm{E} 1$ & E2 & E3 & E4 & E5 & E6 & E7 & E8 & E9 \\
\hline Staurastrum boergensenii var. elegans & & & & & & $x$ & & & \\
\hline S.boergensenii var. scotii & & & & & & $x$ & & & \\
\hline S.brachiatum & $x$ & & & & & $x$ & & $x$ & \\
\hline S.brasiliense var. porrectum & & & $x$ & $x$ & & & & & \\
\hline S.brebissonii var. brasiliense & & & & $x$ & $x$ & & $x$ & & $x$ \\
\hline S.elegantissimum var. brasiliense & $x$ & $x$ & $x$ & $x$ & & & $x$ & & $x$ \\
\hline S.dispergens & $x$ & & & & & & & & \\
\hline S. gladiosum f. ornata & $x$ & & & & & & & & \\
\hline S.grallatorium var. brasiliense & $x$ & & $x$ & & & & & & \\
\hline S.leptacanthum & & $x$ & $x$ & $x$ & & & & & \\
\hline S.novae-cesareae var. brasiliense & $x$ & & & & & & & & \\
\hline S.nudibrachiatum & & & & & & & $x$ & & \\
\hline S.paradoxum & & & & & & & $x$ & & \\
\hline S.quadrangulare var. Iongispinum & & & $x$ & & & & $x$ & $x$ & $x$ \\
\hline S.quadrangulare var. prolificum & & & & & & $x$ & $x$ & & \\
\hline S.rotula & & & & & & & $x$ & & \\
\hline S.sebaldii var. ornatum f. planctonica & & & & & & & $x$ & & \\
\hline S. setigerum var. occidentale & & & & & & & $x$ & & \\
\hline S.setigerum var. pectinatum & & $x$ & & & & $x$ & $x$ & & \\
\hline S. setigerum var. tristichum f. furcatum & $x$ & & & & & & & & \\
\hline S.stelliferum var. stelliferum & & & & & & & & $x$ & $x$ \\
\hline S.tectum var. ayayense & & & & & & & $x$ & & \\
\hline S.trifidum var. inflexum & & & & $x$ & & & $x$ & $x$ & \\
\hline Staurodesmus clepsidra var. obtusus & & & & & & $x$ & & & \\
\hline
\end{tabular}


Tabela 2 - Continuação

\begin{tabular}{|c|c|c|c|c|c|c|c|c|c|}
\hline Espécies/estações de coletas & $\mathrm{E} 1$ & E2 & E3 & E4 & E5 & E6 & E7 & E8 & E9 \\
\hline St.cuspidatus var. groenbladii & & & & & & $x$ & & & \\
\hline St. convergens & $X$ & & $X$ & & & & $X$ & & \\
\hline St. extensus var. extensus & & & & & & $x$ & & & \\
\hline St. glaber var. flexispinus & & & & $X$ & & & & $X$ & \\
\hline St. validus var. subvalidus & $X$ & & & & & & & & \\
\hline St. triangularis var. triangularis & & & & & & & $x$ & & \\
\hline Xanthidium canadense var. magnum & & & & $X$ & & & & & $X$ \\
\hline$X$. fragile & $x$ & & & & & & & & \\
\hline X. mamillosum var. borgei & $X$ & & $X$ & & & $\mathrm{X}$ & & & \\
\hline X. mamillosum var. longispinum & & & & & & $\mathrm{X}$ & & & \\
\hline X. trilobum & & & & & & $x$ & & & \\
\hline
\end{tabular}

\section{AGRADECIMENTOS}

Agradecemos ao Fundo Brasileiro para a Biodiversidade (FUNBIO), Instituto Brasileiro de Meio Ambiente e Recursos Naturais Renováveis (IBAMA-AP) e Fundação CAPES (PRODOC) que financiaram este projeto. Ao MCT-INPA/CNPq pela bolsa DTI concedida ao segundo autor, e à FAPEAM pela bolsa de doutorado da primeira autora. Agradecemos também ao Instituto Estadual de Pesquisas do Amapá (IEPA) e Maxley Barbosa Dias pelo apoio logístico.

\section{BIBLIOGRAFIA CITADA}

Bicudo, C.E.M.; Menezes, M. 2006. Genera of continental algae from Brazil: Identification key and descriptions. Rima: São Carlos, São Paulo. 508 pp.

Brook, A.J. 1981. The biology of desmids. Blackwell Scientific Publications. Oxford, London. 276 pp.

Coesel, P.F.M. 1982. Structural characteristics and adaptations of desmids communities. Journal of Ecology. 70: 163-177.

Coesel, P.F.M. 1996. Biogeography of desmids. Hydrobiologia, 336: 41-53.

Coesel, P.F.M.; Wardenaar, K. 1990. Growth responses of planktonic desmid species in a temperature-light gradient. Freshwater Biology. 23: 551-560.

Croasdale, H.; Flint, E.A.; Racine, M.M. 1994. Flora of New Zealand: Freshwater algae, Chlorophyta, Desmids with ecological comments on their habitats. Volume III: Staurodesmus, Staurastrum and the Filamentosus desmids. Manaaki Whenua Press. Lincoln, Canterbury, New Zealand. 218 pp.

Dias, M.B. 2007. Composition and abundancy of phytoplankton on the southwest of the Lago Piratuba Biological Reserve (Amapá, Brazil). Dissertação de Mestrado, Instituto Nacional de Pesquisas da Amazônia/Fundação Universidade do Amazonas, Manaus, Amazonas. 111 pp. (in Portuguese, with abstract in English).
Förster, K. 1963. Desmids of Brazil, 1: Northern Brazil. Revue Algologique: nouvelle série, 7: 38-92.( In German)

Förster, K. 1964. Desmids of Brazil, 2: Bahia, Goyaz, Piauhy and Northern Brazil. Hydrobiologia, 23: 321- 505. (in German)

Förster, K. 1969. Amazonian Desmids. 1st part. Area Santarém. Amazoniana, 2: 5-232. (in German with abstract in Portuguese).

Förster, K. 1974. Amazonian Desmids. 2nd part. Area MauésAbacaxis. Amazoniana, 5(2): 135-242. (in German with abstract in Portuguese).

Gerrath, J.F. 1993. The biology of desmids: a decade of progress. p. 79-192. In Round, F. E.; Chapman D. J. Progress in phycological research 9. Biopress, Bristol. 377 pp.

Gerrath, J.F. 2003. Conjugating green algae and desmids, p. 353-382. In: Wehr, J.D.; Sheath, R.G. Freshwater algae of North America: ecology and classification. Elsevier Science Academic Press. 507 pp.

Grönblad, R. 1945. Brazilian algae, specially Desmidiaceae from low region of Amazon river from August Ginzberger samples on year of MCMXXVII. Acta Societatis Scientiarum Fennicae, Nova Séries B, 2: 1-42 (in Latin).

Happey-Wood, C.M. 1988 Ecology of freshwater planktonic green algae, p. 175-226. In: Sandgren, C.D. Growth and reproductive strategies of freshwater phytoplankton. Cambridge University Press. 443 pp.

Lopes, M.R.M.; Bicudo, C.E.M. 2003. Desmid Flora of the Flood Plain Lake, Acre River, Amazonas State, Brazil. Acta Amazonica, 33: 167-212. (in Portuguese, with abstract in English).

Melo, S.; Rebelo S.R.M.; Souza, K.F.; Soares C.C. Sophia, M.G. 2005. Desmids with planktonic occurrence, p. 99-108. In: Santos Silva, E.N.; Aprile, F.M.; Scudeller, V.V.; Melo, S. Biotupé: physical environment, biological and sociocultural diversity of the low Negro river, Central Amazon. Instituto Nacional de Pesquisas da Amazônia, Manaus, Amazonas. (in Portuguese).

Melo, S.; Souza, K.F. 2009. Annual and interannual fluctuation of desmids species in a black water Amazon floodplain lake (Lago Cutiuaú, Amazonas State, Brazil). Acta Scientiarum-Biological Science, 31: 235-243 (in Portuguese, with abstract in English). 


\section{ACTA}

Mix, M. 1972. The fine structure of Mesotaeniaceae and Gonatozygaceae with a comparison of the wall types of the Conjugatophyceae and over their systematic value. Archivie für Mikrobiologie, 81: 197-220 (in German).

Scott, A.M., Grönblad, R.; Croasdale, H.T. 1965. Desmids from the Amazon Basin, Brazil, collected by Dr. H. Sioli. Acta Botannica Fennica, 69: 3-93.

Silveira, O.F.M. 1998. The coastal plain of Amapá: dynamic coastal environmental influenced by large river sources quaternary. Tese de doutorado, Universidade Federal do Pará, Belém. 215 pp. (in Portuguese, with abstract in English).
Sophia, M.G.; Huszar, V.L.M. 1996. Planktonic desmids of three Amazonian systems (Lake Batata, Lake Mussurá e Trombetas River), Pará, Brasil. Amazoniana, 14: 75-90.

Teiling, E. 1967. The desmid genus Staurodesmus: a taxonomic study. Arkiv för Botanik, 6: 467-629.

Thomasson, K. 1971. Amazonian algae. Mémoires de l'Institute Royale des Sciences Naturelles de Bélgique 10, 86: 1-57.

Recebido em 05/01/2010

Aceito em 07/12/2010 